Fellowship. We are indebted to Dr. Y.-K. Kim for helpful conversations and his critical reading of the manuscript, and to Dr. S. T. Manson for communicating his unpublished results.

*Work performed under the auspices of the U.S. Atomic Energy Commission.

${ }^{1}$ U. Fano and J. W. Cooper, Rev. Mod. Phys. 40, 441 (1968).

${ }^{2}$ F. J. Comes and H. G. Sälzer, Z. Naturforsch. 19a, 1230 (1964).

${ }^{3}$ K. T. Lu, Phys. Rev. A $\underline{4}, 579$ (1971).

${ }^{4}$ J. A. R. Samson and R. B. Cairns, Phys. Rev. 173, 80 (1968).

${ }^{5}$ D. Liberman, J. T. Waber, and D. T. Cromer, Phys. Rev. 137, 27 (1965).

${ }^{6}$ J. C. Slater, Advan. Quant. Chem. 6 , 1 (1972).
${ }^{7} \mathrm{~J}$. Berkowitz, J. Chem. Phys. $\underline{56}, 2766$ (1972).

${ }^{8}$ J. W. Cooper, Phys. Rev. 128, 681 (1962).

${ }^{9}$ H. Harrison, R. I. Schoen, R. B. Cairns, and K. E. Schubert, J. Chem. Phys. 50, 3930 (1969).

${ }^{10}$ R. B. Cairns, H. Harrison, and R. I. Schoen, J. Chem. Phys. $\underline{51}, 5440$ (1969).

${ }^{11}$ R. B. Cairns, H. Harrison, and R. I. Schoen, J. Chem. Phys. 53, 96 (1970).

${ }^{12}$ J. A. R. Samson, Advan. At. Mol. Phys. 2, 177

(1966), and references therein.

${ }^{13}$ J. L. Dehmer, J. Berkowitz, and Y.-K. Kim, Proceedings of the Eighth International Conference on the Physics of Electronic and Atomic Collisions, Belgrade, Yugoslavia, 16-20 July 1973, Abstracts of Papers (to be published).

${ }^{14}$ A. R. P. Rau and U. Fano, Phys. Rev. A $\underline{4}, 1751$ (1971).

${ }^{15} \mathrm{~S}$. T. Manson, private communication.

${ }^{16}$ K. T. Lu and U. Fano, Phys. Rev. A 2, 81 (1970);

C. M. Lee and K. T. Lu, Phys. Rev, A (to be published).

\title{
Thomas-Fermi Theory Revisited
}

\author{
Elliott H. Lieb* \\ Institut des Hautes Etudes Scientifiques, 91440-Bures-sur-Yvette, France \\ and \\ Barry Simont \\ Department of Physics, Eidgenössische Technische Hochschule, CH-8049 Zurich, Switzerland
}

(Received 8 June 1973)

\begin{abstract}
We show that the Thomas-Fermi theory is exact for atoms, molecules, and solids as $Z \rightarrow \infty$.
\end{abstract}

The Thomas-Fermi (TF) theory of atoms and molecules ${ }^{1}$ is now more than 45 years old. The literature on the subject is vast ${ }^{2}$ yet there remain more than a few unresolved problems both of principle and interpretation. Can one show that there is an electron density function $\rho$ which actually minimizes the TF energy expression and that it satisfies the TF equation? Does this $\rho$ represent the true electron density as computed from the Schrödinger equation as $Z \rightarrow \infty$ ? If so, there appear to be some "paradoxes": For atoms the density falls off exponentially with distance, while in TF theory ${ }^{3}$ it falls off as $\gamma^{-6}$; in TF theory atoms shrink in size as $Z^{-1 / 3}$ instead of growing; the electron density in TF theory is infinite at the nuclei instead of being finite; in TF theory molecules never bind. ${ }^{4}$

Recently, considerable progress has been made in showing that TF theory is applicable to high- density matter ${ }^{5}$ but the questions raised above are of a different nature, especially in the fact that a parameter in the problem, $Z$, becomes infinite; it is that which causes the electron density to become infinite. We report here the results of our analysis ${ }^{6}$ of the above questions, and the main conclusion is that TF theory, when correctly interpreted, is rigorously exact as $Z \rightarrow \infty$.

We also show that TF theory is rigorously exact for solids in this limit and leads to a periodic $\rho$ which satisfies the TF equation with the periodic Coulomb potential. This $Z \rightarrow \infty$ limit is related to, but is not the same as, the high-density limit with fixed $Z$, a case to which TF theory is often applied. ${ }^{7}$ We make no statements about this latter situation.

The TF energy functional in the presence of $k$ nuclei of positive charges and positions $\left(z_{i}, R_{i}\right)$, $i=1, \ldots, k$, in units such that $h^{2}(3 / 8 \pi)^{2 / 3}(2 m)^{-1}=1$ and $|e|=1$, is

$$
E\left(\rho ; z_{1}, \ldots, z_{k} ; R_{1}, \ldots, R_{k}\right)=\frac{3}{5} \int \rho(x)^{5 / 3} d^{3} x+\frac{1}{2} \iint \rho(x) \rho(y)|x-y|^{-1} d^{3} x d^{3} y-\int \rho(x) \sum_{i=1}^{k} z_{i}\left|x-R_{i}\right|^{-1} d^{3} x
$$


The electric potential is defined to be

$$
\varphi(x)=-\int \rho(y)|x-y|^{-1} d^{3} y+\sum_{i=1}^{k} z_{i}\left|x-R_{i}\right|^{-1} .
$$

The subsidiary condition is that $\rho(x) \geqslant 0$ and

$$
\lambda \equiv \int \rho(x) d^{3} x=\text { electron number, }
$$

but we may consider $\lambda$ to be an arbitrary constant. Finally,

$$
Z=\sum_{i=1}^{k} z_{i}
$$

The cases $\lambda=Z, \lambda<Z$, and $\lambda>Z$ are the neutralmolecule, the positive-ion, and the negative-ion cases, respectively. While it is easy to $\mathrm{see}^{8}$ that for a given $\lambda, E(\rho)$ is bounded below and is strictly convex in $\rho$, the crucial question is whether there is a $\rho$ that actually minimizes $E$ and whether it is unique. For all $\lambda \geqslant 0$ we define $E_{\lambda}$ to be the infimum of (1) under condition (3).

Theorem 1: (a) If $\lambda \leqslant Z, E$ has a unique minimizing $\rho$. If $\lambda>Z$ there is no such $\rho . E_{\lambda}$ is convex and monotone decreasing, and $E_{\lambda}=E_{Z}$ for $\lambda \geqslant Z$. (b) If $\lambda=Z, \rho(x) \rightarrow 1728|x|^{-6}$ as $|x| \rightarrow \infty, \rho(x)$ $>0$ for all $x, \rho(x)$ is $C^{\infty}$ away from the nuclei, and $\rho(x)^{2 / 3}=\varphi(x)$. (c) If $\lambda<Z, \rho(x)$ has compact support and is $C^{\infty}$ where $\rho>0 ; \rho(x)$ is $C^{1}$ and $\varphi(x)$ is $C^{2}$ everywhere. There is a $C>0$ such that $\rho(x)^{2 / 3}$ $=\varphi(x)-C$ when $\varphi(x) \geqslant C$, and $\rho(x)=0$ when $\varphi(x)$ $\leqslant C$. In particular, $\varphi$ satisfies the TF differential equation. (d) The constant $C$ above is the negative of the chemical potential (Fermi energy), i.e., $d \boldsymbol{E}_{\lambda} / d \lambda=-C$. (e) $\varphi(x) \rightarrow z_{i}\left|x-R_{i}\right|^{-1}+$ const as $x \rightarrow R_{i}$. Hence $\rho(x) \sim z_{i}{ }^{3 / 2}\left|x-R_{i}\right|^{-3 / 2}$ near $R_{i}$.

The next two theorems refer to the neutral case and we denote the minimizing $\rho$ by $\rho\left(x ; z_{1}, \ldots, z_{k}\right.$; $R_{1}, \ldots, R_{k}$ ). The TF energy plus the nuclear Coulomb energy,

$$
E_{Z}+\frac{1}{2} \sum_{i \neq j}^{k} z_{i} z_{j}\left|R_{i}-R_{j}\right|^{-1},
$$

will be denoted by $E\left(z_{1}, \ldots, z_{k} ; R_{1}, \ldots, R_{k}\right)$. The following result ${ }^{9}$ is due to Teller ${ }^{4}$ and is important in the proofs of Theorems 1 and 3 .

Theorem 2: (a) If $R_{1}, \ldots, R_{k}$ are fixed and $z_{i}{ }^{*}$ $\geqslant z_{i}, i=1, \ldots, k$ with some $z_{j}{ }^{*}>z_{j}$, then $\rho\left(x ; z^{*} ; R\right)$ $>\rho(x ; z ; R)$ for all $x$. Moreover, if $z_{i}{ }^{*}=z_{i}$ then

$$
\lim _{x \rightarrow R_{i}} \rho\left(x ; z^{*} ; R\right)-\rho(x ; z ; R)
$$

exists and is strictly positive. (b) Molecules never bind in TF theory, i.e.,

$$
\begin{gathered}
E\left(z_{1}, \ldots, z_{n+k} ; R_{1}, \ldots, R_{n+k}\right)>E\left(z_{1}, \ldots, z_{k} ; R_{1}, \ldots, R_{k}\right) \\
+E\left(z_{k+1}, \ldots, z_{n+k} ; R_{k+1}, \ldots, R_{n+k}\right)
\end{gathered}
$$

The following theorem about the thermodynamic limit for solids in TF theory holds for arbitrary Bravais lattices with any distribution of nuclei in a unit cell, but for simplicity we state it for a simple cubic lattice.

Theorem 3: Let $z$ be fixed. For each subset $\Lambda$ of the lattice of integral points, let $\rho_{\Lambda}$ denote the solution of the neutral TF theory with nuclei of charge $z$ at each point of $\Lambda$, and let $E_{\Lambda}$ be the TF energy. Then (a) as $\Lambda \rightarrow \infty$ in the sense of van Hove, $\rho_{\Lambda}(x)$ converges pointwise to a function $\rho(x)$ and $E_{\Lambda} /|\Lambda|$ converges to an intensive energy $e$. (b) $\rho$ is periodic with unit period. (c) $\rho$ is the solution to the neutral $\mathrm{TF}$ problem in a unit cell in which $|x|^{-1}$ is replaced by the periodic ${ }^{10} \mathrm{Cou}-$ lomb potential $G_{p}(x)$. $e$ is the corresponding $\mathrm{TF}$ energy.

Unlike the neutral-molecule case, the Lagrange multiplier $C$ will be negative for solids; it is related to the compressibility. The basic tool in the proof of Theorem 3 is Theorem 2, e.g., (5) implies that $E_{\Lambda}$ is superadditive in $\Lambda$.

We turn next to the question of how TF theory is related to the solution of Schrödinger's equa tion. We first note that TF theory has a simple scaling relation: Let $E_{N}\left[\rho_{N}(x)\right]$ denote the TF energy [function] for $k$ nuclei of charges and positions $z_{i} N, R_{i} N^{-1 / 3}(i=1, \ldots, k)$, and $\int \rho_{N}(x) d^{3} x$ $=\lambda N$, with $\lambda \leqslant Z=\sum z_{i}$. Then

$$
E_{N}=N^{7 / 3} E_{1}, \quad \rho_{N}(x)=N^{2} \rho_{1}\left(N^{1 / 3} x\right) .
$$

This relation allows us to relate the quantum problem for large $N$ (electron number) to an $N$ independent TF problem.

Theorem 4: For $\lambda \leqslant Z$, let $E_{N}{ }^{0}$ and $\rho_{N}{ }^{0}(x)$ denote the ground-state energy and one-electron distribution function for $N$ spin- $\frac{1}{2}$ electrons obeying the Pauli principle and interacting with $k$ nuclei as described above. Then (a) $N^{-7 / 3} E_{N}{ }^{0}-E_{1}$ as $N \rightarrow \infty$; (b) $N^{-2} \rho_{N}{ }^{0}\left(N^{-1 / 3} x\right) \rightarrow \rho_{1}(x)$ as $N \rightarrow \infty$, where convergence in (b) means that for any domain $D \subset R^{3}$, the expected fraction of electrons in $N^{-1 / 3} D$ approaches $\int_{D} \rho_{1}(x) d^{3} x$.

The methods employed to prove Theorems 1 , 2 , and 3 include $L^{p}$ space techniques, the theory of convex functions, and the theory of harmonic functions. The basic fact used in Theorem 4 is that the introduction of Neumann (Dirichlet) boundary conditions on subdomains lowers (raises) the ground-state energy. This fact has been used before. ${ }^{11}$ In two places problems arise that do not appear in the theory of gravitating fermions. ${ }^{5,12}$

From the above theorems a picture of large $-Z$ 
atoms that resolves the aforementioned "paradoxes" can be formulated. The electron cloud is divided into five regions:

(1) An inner core of size $\sim Z^{-1 / 3}$ described by $\mathrm{TF}$ theory in which the density is $\sim Z^{2}$ and in which there are $\sim Z$ electrons.

(2) The mantle of the core in which $\rho \sim 1728|x|^{-6}$ independently of $Z$. The length scale of the mantle is also $Z^{-1 / 3}$ and the core and the mantle contain $100 \%$ of the electrons as $Z \rightarrow \infty$.

(3) A complicated intermediate region.

(4) The outer shells. Crude models, in which one takes into account screening, suggest that this region has a size of order 1 and contains $\sim Z^{2 / 3}$ electrons. Chemistry takes place here.

(5) The outside of the atom where the density falls off exponentially with distance.

In understanding TF theory, then, one principle must be borne in mind: TF theory describes the atomic core and mantle, and only those. These two regions contain almost all the electrons, but their size shrinks like $Z^{-1 / 3}$. There is no difficulty reconciling the $|x|^{-6}$ falloff here with the exponential falloff in region (5). The shell region, which is what one sees chemically, is enormously large compared to the $\mathrm{TF}$ region but it contains a negligible fraction of electrons. It is not surprising that molecules fail to bind in $\mathrm{TF}$ theory, because to do so would require core binding and, as Teller noted, ${ }^{4}$ this would imply that molecular sizes would shrink as $Z^{-1 / 3}$ and binding energies would grow as $Z^{7 / 3}$. Instead, binding occurs in the shell region.

The authors thank Dr. N. Kuiper for the hospitality of the Institut des Hautes Etudes Scientifique. We are also indebted to Dr. J. Barnes for suggesting this problem and for his encouragement and to Dr. H. Kunz for useful discussions.

*Work partially supported by National Science Foundation Grant No. GP-31674X and by a Guggenheim Memorial Foundation Fellowship. Permanent address: Department of Mathematics, Massachusetts Institute of Technology, Cambridge, Mass. 02139.

$\dagger$ Alfred P. Sloan Foundation Fellow. Permanent ad- dress: Department of Mathematics and Department of Physics, Princeton University, Princeton, N.J. 08540.

${ }^{1}$ The original theory appears in L. H. Thomas, Proc. Cambridge Phil. Soc. 23, 542 (1927); E. Fermi, Atti Reale Accad. Naz. Lincei, Rend., C1. Sci. Fis., Mat., Natur. $\underline{6}, 602$ (1927).

${ }^{2}$ For reviews of the literature, see P. Gombás, Die Statistische Theorie des Atoms (Springer, Berlin, 1949); N. H. March, Advan. Phys. $\underline{6}, 21$ (1957).

${ }^{3}$ A. Sommerfeld, Z. Phys. 78, 283 (1932). For the central case (atoms), the $r^{-6}$ behavior has recently been rigorously established by E. Hille, J. Anal. Math. $\underline{23}, 147$ (1970).

${ }^{4}$ E. Teller, Rev. Mod. Phys. 34, 627 (1962). See also N. Balazs, Phys. Rev. 156, 156 (1967); J. W. Sheldon, Phys. Rev. 99, 1291 (1955).

${ }^{5} \mathrm{P}$. Hertel and W. Thirring, Commun. Math. Phys. 24, 22 (1971); P. Hertel, H. Narnhofer, and W. Thirring, Commun. Math. Phys. 28, 159 (1972).

${ }^{6}$ E. Lieb and B. Simon, "The Thomas-Fermi Theory of Atoms and Molecules" and "The Thomas-Fermi Theory of Solids" (to be published).

${ }^{7}$ J. F. Barner, Phys. Rev. 153, 269 (1967); R. F. Trunin, M. A. Podurets, G. V. Simakov, L. V. Popov, and B. N. Moiseev, Zh. Eksp. Teor. Fiz. 62, 1043 (1972) [Sov. Phys. JETP 35, 550 (1972)].

${ }^{8}$ In order for $E(\rho)$ to be well defined, we demand that $\rho$ be positive with $\int \rho d x<\infty$ and $\int \rho^{5 / 3} d x<\infty$. The boundedness follows from Hölder's inequality.

${ }^{9}$ Some concern has been expressed about the role of the self-energy in Teller's proof; this has led to some questioning of the validity of his results. For this reason, we have provided a rigorous proof of Teller's results (see Ref. 6). We emphasize that the basic idea in our proof is Teller's. Because $L^{p}$ techniques allow us to handle an unsmeared nuclear charge, our proof is somewhat more direct then Teller's and no self-energies enter.

${ }^{10} G_{p}$ is a periodic solution of $-\nabla^{2} G_{p}(x)=\delta(x)-1$. While such a solution is only unique up to a constant, the addition of a constant to $G_{p}$ does not affect the TF energy because of neutrality.

${ }^{11}$ It has been used to provide the validity of the Van der der Waals theory in the limit of long-range forces in E. Lieb, J. Math. Phys. (N.Y.) 7, 1016 (1966), and to prove that WKB asymptotically counts the number of bound states in the strong-coupling limit in A. Martin, Helv. Phys. Acta $\underline{45}, 140$ (1972).

${ }^{12}$ In Theorem 1, we must show that charge in the Thomas-Fermi theory does not leak out at infinity-it is for this reason that the restriction $\lambda \leqslant Z$ enters. In Theorem 4 , we must show that the electron cloud does not collapse into the nucleus at a rate faster than $Z^{-1 / 3}$. 